\title{
Lie symmetry Analysis and Invariant Solutions for Multiregion Neutron Diffusion Equation
}

\author{
Rakotondravanona Jean Eric, Raboanary Roland \\ Department of Physics \& Applications, University of Antananarivo, Antananarivo, Madagascar \\ Email address: \\ jrakotondravanona@yahoo.com (R. J. Eric)
}

\section{To cite this article:}

Rakotondravanona Jean Eric, Raboanary Roland. Lie symmetry Analysis and Invariant Solutions for Multiregion Neutron Diffusion Equation. World Journal of Applied Physics. Vol. 3, No. 2, 2018, pp. 25-33. doi: 10.11648/j.wjap.20180302.12

Received: May 19, 2018; Accepted: June 6, 2018; Published: July 7, 2018

\begin{abstract}
In this paper, an approach of determining analytical solutions of the mono-kinetic multiregion neutron diffusion equation from two-dimensional Cartesian geometry is presented. The technical approach is based on the Lie symmetry group for partial differential equation. The local symmetry groups to the one-parameter transformation are obtained. The invariant solutions spanned of an expansion of neutron fluxes with respect to the space, time and material regions are reported.
\end{abstract}

Keywords: Multiregion Neutron Diffusion Equation, Symmetry Groups, Invariant Solutions

\section{Introduction}

The mathematical description of neutron diffusion for $m$ regions is depended on time $t$, space $\vec{r}$ and media. This description is provided by the neutron diffusion theory (Fick's law), necessary to the comprehension of many properties of the nuclear reactor physics.

Neutron diffusion is performed on a region towards other regions. Indeed, the diffusion theory is used to define the neutron's behavior. The neutron flux is then a combination of time, space and media.
The flux and the current are continuous through the system conditions after the change of region. The flux and the current are continuous through the system conditions after the change of region. The fluctuation varies along the direction $\vec{r}$ and this one can be applied by coupling flux and homogeneous regions $m=1,2, \ldots M$. A region is considered by the material which it is composed (fuel, control rod,...).

The homogeneous mono-kinetic multiregion neutron diffusion equation (MNDE) for multiplying media is, assumed without delayed neutrons, external source and feedback mechanisms and other influences, written as

$$
\frac{1}{v_{m}} \frac{\partial}{\partial t} \Phi_{m}(\vec{r}, t)=D_{m} \nabla^{2} \Phi_{m}(\vec{r}, t)+\left(v_{m} \Sigma_{f m}-\Sigma_{a m}\right) \Phi_{m}(\vec{r}, t),
$$

where $t>0, \Phi_{m}(\vec{r}, t)\left(n \cdot m^{-2} \cdot s^{-1}\right), v_{m}\left(m \cdot s^{-1}\right), D_{m}(m)$, $\Sigma_{a m}\left(m^{-1}\right), \Sigma_{f m}\left(m^{-1}\right)$ and $v_{m}\left(\right.$ n. fission $\left.{ }^{-1}\right)$ are denoted the neutron flux, neutron speed, diffusion coefficient, macroscopic absorption cross section, macroscopic fission cross section and the fraction of neutrons emitted by fission, respectively [1-5], [14]. The model of analytic solutions to the diffusion equation is appropriated for the conditions of prompt neutron behavior.

The Lie symmetry group is established to the variable (dependent or independent) transformations leaving partial differential equation (PDE) invariant [6-8], [11]. The key to find symmetry groups is the group related to the parameter $\varepsilon \ll 1$. Using Lie symmetry group allow to determine also the invariant solutions to the mono-kinetic MNDE.
The objective of the paper is to obtain symmetry groups and invariant solutions for the MNDE using the Lie symmetry group. The outline is as follows. The Section 2 considers the material. The section 3 presents the Lie symmetry analysis of the MNDE. The Section 4 may obtain invariant solutions of the equation. Finally, in Section 5, conclusions are made.

\section{Material}

In this section, the Eq. (1) is given from Cartesian geometry $(x, y)$ related to the expression of the mono-kinetic neutron flux $[12,13]$. Each coefficient is constant within a homogeneous region. In two-dimensional, the equation is presented, extending over the interval $x_{m-1}<x<x_{m}\left(x_{0}=\right.$ 
$0), y_{m-1}<y<y_{m}\left(y_{0}=0\right)$ for $m=1,2, \ldots M$, as

$$
\Phi_{m, t}=a_{m}\left(\Phi_{m, x x}+\Phi_{m, y y}\right)+b_{m} \Phi_{m}(x, y, t)
$$

with

$$
v_{m} D_{m}=a_{m}
$$

and

$$
v_{m}\left(v_{m} \Sigma_{f m}-\Sigma_{a m}\right)=b_{m} .
$$

The 2D BIBLIS benchmark is used as a $2 \mathrm{D}$ representative problem to the parametrical statement of neutron coefficients $[9,10],[12,13]$. The BIBLIS core uses a checkerboard pattern, formed by fuel elements and control bar. Fuel assemblies have widths of $x_{m}=y_{m}=23.1226 \mathrm{~cm}$.

The seven different compositions of material regions are presented in the core and surrounded by reflector of width $x_{m}=y_{m}=23.1226 \mathrm{~cm}$.

The solutions of diffusion equation will be illustrated from physical parameters relating $m=8$ to regions of BIBLIS benchmark.

Table 1. BIBLIS 2D-Benchmark of macroscopic cross sections for the group of fast neutron.

\begin{tabular}{llll}
\hline $\boldsymbol{m}$ & $\boldsymbol{D}_{\boldsymbol{m}}(\boldsymbol{m})$ & $\boldsymbol{\Sigma}_{\boldsymbol{a m}}\left(\boldsymbol{m}^{-\mathbf{1}}\right)$ & $\boldsymbol{\Sigma}_{\boldsymbol{f m}}\left(\boldsymbol{m}^{\mathbf{- 1}}\right)$ \\
\hline 1 & $1.4360 \times 10-2$ & 0.95042 & 0.58708 \\
2 & $1.4366 \times 10^{-2}$ & 0.96785 & 0.61908 \\
3 & $1.3200 \times 10^{-2}$ & 0.26562 & 0.00000 \\
4 & $1.4389 \times 10^{-2}$ & 1.03630 & 0.74527 \\
5 & $1.4381 \times 10^{-2}$ & 1.00030 & 0.61908 \\
6 & $1.4385 \times 10^{-2}$ & 1.01320 & 0.64285 \\
7 & $1.4389 \times 10^{-2}$ & 1.01650 & 0.61908 \\
8 & $1.4393 \times 10^{-2}$ & 1.02940 & 0.64285 \\
\hline
\end{tabular}

The fraction of neutrons emitted by fission is $v_{m}=2.47$. The neutron speed is assumed to $v_{m}=3 \times 10^{5} \mathrm{~m} . \mathrm{s}^{-1}$ and the initial flux $\Phi_{m}^{0}=10^{3} n \cdot m^{-2} s^{-1}[5]$.

The coefficients $a_{m}$ and $b_{m}$ of system are:

Table 2. The values of the coefficients $a_{m}$ and $b_{m}$.

\begin{tabular}{lll}
\hline Region & $\boldsymbol{a}_{\boldsymbol{m}}\left(\boldsymbol{m}^{\mathbf{2}} \cdot \boldsymbol{s}^{-\mathbf{1}}\right)$ & $\boldsymbol{b}_{\boldsymbol{m}}\left(\boldsymbol{s}^{\mathbf{- 1}}\right)$ \\
\hline 1 & $4.3060 \times 10^{3}$ & $-1.09002 \times 10^{5}$ \\
2 & $4.3098 \times 10^{3}$ & $-1.04631 \times 10^{5}$ \\
3 & $3.9600 \times 10^{3}$ & $-0.79686 \times 10^{5}$ \\
4 & $4.3167 \times 10^{3}$ & $-0.87309 \times 10^{5}$ \\
5 & $4.3143 \times 10^{3}$ & $-1.14366 \times 10^{5}$ \\
6 & $4.3155 \times 10^{3}$ & $-1.11105 \times 10^{5}$ \\
7 & $4.3167 \times 10^{3}$ & $-1.19226 \times 10^{5}$ \\
8 & $4.3179 \times 10^{3}$ & $-1.15965 \times 10^{5}$ \\
\hline
\end{tabular}

The boundary conditions at the surface, the continuity

$$
X=\tau\left(t, x, y, \Phi_{m}\right) \frac{\partial}{\partial t}+\xi\left(t, x, y, \Phi_{m}\right) \frac{\partial}{\partial x}+\eta\left(t, x, y, \Phi_{m}\right) \frac{\partial}{\partial y}+\zeta\left(t, x, y, \Phi_{m}\right) \frac{\partial}{\partial \Phi_{m}} .
$$

The second prolongation $X^{(2)}$ formula gives

$$
\begin{gathered}
\left.\operatorname{Pr}^{(2)} X(\Delta)\right|_{\Delta=0}=0 \rightarrow X^{(2)}(\Delta)=0, \\
X^{(2)}=X+\zeta_{t} \frac{\partial}{\partial \Phi_{m, t}}+\zeta_{x x} \frac{\partial}{\partial \Phi_{m, x x}}+\zeta_{y y} \frac{\partial}{\partial \Phi_{m, y y}},
\end{gathered}
$$

where the components $\zeta_{t}, \zeta_{x x}$ and $\zeta_{y y}$ are the coefficient conditions within each interface and the initial condition for time-dependent problem are defined to the diffusion equation resolution with the geometrical description and physical environments.

Within time-dependent situation, spatial distribution of neutron at $t=0$ is:

$$
\Phi_{m}(x, y, 0)=\Phi_{m}^{0}
$$

so that the initial flux is constant within each region and considered as the initial state.

The interface flux continuity condition is

$$
\Phi_{m}(x, y, t)=\Phi_{m+1}(x, y, t),
$$

while that for the current is

$$
-\left.D_{m} \frac{\partial \Phi_{m}(x, y, t)}{\partial(x, y)}\right|_{x=x_{m}} ^{y=y_{m}}=-\left.D_{m+1} \frac{\partial \Phi_{m+1}(x, y, t)}{\partial(x, y)}\right|_{x=x_{m}} ^{y=y_{m}}
$$

for $m=1,2 \ldots M-1$.

At the surface, a variety of conditions can be imposed depending on the application. The boundary conditions are

$$
\begin{gathered}
\lim _{(x, y) \rightarrow+\infty} \Phi_{m}(x, y, t)=\lim _{(x, y) \rightarrow 0} \Phi_{m}(x, y, t)=0 \\
\lim _{(x, y) \rightarrow+\infty} \frac{\partial \Phi_{m}(x, y, t)}{\partial(x, y)}=\lim _{(x, y) \rightarrow 0} \frac{\partial \Phi_{m}(x, y, t)}{\partial(x, y)}=0 .
\end{gathered}
$$

These conditions materialize the limit of absorbent material at the core edge, including reflector.

\section{Lie Symmetry Analysis}

The system is described as

$$
\Delta\left(t, x, y, \Phi_{m}, \Phi_{m, t}, \Phi_{m, x x}, \Phi_{m, y y}\right)=0 .
$$

Let us consider one-parameter Lie group of transformations:

$$
\begin{gathered}
\tilde{t}=t+\varepsilon \tau\left(t, x, y, \Phi_{m}\right)+\mathcal{O}\left(\varepsilon^{2}\right), \\
\tilde{x}=x+\varepsilon \xi\left(t, x, y, \Phi_{m}\right)+\mathcal{O}\left(\varepsilon^{2}\right), \\
\tilde{y}=y+\varepsilon \eta\left(t, x, y, \Phi_{m}\right)+\mathcal{O}\left(\varepsilon^{2}\right), \\
\widetilde{\Phi}_{m}=\Phi_{m}+\varepsilon \zeta\left(t, x, y, \Phi_{m}\right)+\mathcal{O}\left(\varepsilon^{2}\right),
\end{gathered}
$$

where $\varepsilon$ is a parameter of group $G$ [6-8]. The components $\tau\left(t, x, y, \Phi_{m}\right), \xi\left(t, x, y, \Phi_{m}\right), \eta\left(t, x, y, \Phi_{m}\right)$ and $\zeta\left(t, x, y, \Phi_{m}\right)$ are the coefficient functions. The vector field associated with the above group of transformations is functions of $X^{(2)}$. The relation (14) gives

$$
X^{(2)}\left(\Phi_{m, t}-a_{m}\left(\Phi_{m, x x}+\Phi_{m, y y}\right)-b_{m} \Phi_{m}=0\right),
$$

therefore, the determining function is

$$
\zeta_{t}-a_{m}\left(\zeta_{x x}+\zeta_{y y}\right)-b_{m} \zeta=0,
$$


with

$$
\begin{aligned}
\zeta_{t}= & D_{t} \zeta-\Phi_{m, t} D_{t} \tau-\Phi_{m, x} D_{t} \xi-\Phi_{m, y} D_{t} \eta \\
\zeta_{x x} & =D_{x}^{2} \zeta-\Phi_{m, x} D_{x}^{2} \xi-\Phi_{m, y} D_{x}^{2} \eta-\Phi_{m, t} D_{x}^{2} \tau \\
& -2 \Phi_{m, x x} D_{x} \xi-2 \Phi_{m, x y} D_{x} \eta-2 \Phi_{m, x t} D_{x} \tau
\end{aligned}
$$

$$
\begin{gathered}
\zeta_{y y}=D_{y}^{2} \zeta-\Phi_{m, x} D_{y}^{2} \xi-\Phi_{m, y} D_{y}^{2} \eta-\Phi_{m, t} D_{y}^{2} \tau \\
-2 \Phi_{m, y x} D_{y} \xi-2 \Phi_{m, y y} D_{y} \eta-2 \Phi_{m, y t} D_{y} \tau
\end{gathered}
$$

where $D_{x}, D_{y}, D_{t}$ are the total derivatives with respect to $x, y$ and $t$ :

$$
\begin{aligned}
D_{t} & =\frac{\partial}{\partial t}+\Phi_{m, t} \frac{\partial}{\partial \Phi_{m}}+\Phi_{m, t x} \frac{\partial}{\partial \Phi_{m, x}}+\Phi_{m, t y} \frac{\partial}{\partial \Phi_{m, y}}+\Phi_{m, t t} \frac{\partial}{\partial \Phi_{m, t}}+\ldots, \\
D_{x} & =\frac{\partial}{\partial x}+\Phi_{m, x} \frac{\partial}{\partial \Phi_{m}}+\Phi_{m, x x} \frac{\partial}{\partial \Phi_{m, x}}+\Phi_{m, x y} \frac{\partial}{\partial \Phi_{m, y}}+\Phi_{m, x t} \frac{\partial}{\partial \Phi_{m, t}}+\ldots, \\
D_{y} & =\frac{\partial}{\partial y}+\Phi_{m, y} \frac{\partial}{\partial \Phi_{m}}+\Phi_{m, y x} \frac{\partial}{\partial \Phi_{m, x}}+\Phi_{m, y y} \frac{\partial}{\partial \Phi_{m, y}}+\Phi_{m, y t} \frac{\partial}{\partial \Phi_{m, t}}+\ldots
\end{aligned}
$$

Substituting relations (18)-(20) into the relation (17) by replacing all $\Phi_{m, t}$ term by $a_{m}\left(\Phi_{m, x x}+\Phi_{m, y y}\right)+b_{m} \Phi_{m}$. By equating the coefficients of the various monomials, the partial derivative orders and various powers of $\Phi_{m}$ to zero, the calculation gives the determining equations of MNDE to the form of the coefficient functions:

$$
\begin{gathered}
\tau_{\Phi_{m}}=\tau_{x}=\tau_{y}=0, \\
\tau_{\Phi_{m} \Phi_{m}}=\tau_{x x}=\tau_{y y}=\tau_{x \Phi_{m}}=\tau_{y \Phi_{m}}=0, \\
\xi_{\Phi_{m}}=\eta_{\Phi_{m}}=0 \Rightarrow \xi_{\Phi_{m} \Phi_{m}}=\eta_{\Phi_{m} \Phi_{m}}=0, \\
-\tau_{t}+2 \xi_{x}=0,-\tau_{t}+2 \eta_{y}=0 \Rightarrow \xi_{y}+\eta_{x}=0, \\
\xi_{x}-\eta_{y}=0,
\end{gathered}
$$$$
\zeta\left(t, x, y, \Phi_{m}\right)=\left(\left(b_{m} \frac{t^{2}}{2}-\frac{t}{2}-\frac{\left(x^{2}+y^{2}\right)}{8 a_{m}}\right) C_{9}-\frac{x}{2 a_{m}} C_{6}-\frac{y}{2 a_{m}} C_{7}+b_{m} t C_{8}+C_{5}\right) \Phi_{m}+\varpi .
$$

$$
\zeta_{\Phi_{m} \Phi_{m}}=0
$$$$
a_{m}\left(\xi_{y y}-\xi_{x x}-2 \zeta_{x \Phi_{m}}\right)-\xi_{t}=0,
$$$$
a_{m}\left(\eta_{x x}-\eta_{y y}-2 \zeta_{y \Phi_{m}}\right)-\eta_{t}=0 \text {, }
$$$$
\zeta_{t}-a_{m}\left(\zeta_{x x}+\zeta_{y y}\right)-b_{m} \zeta+b_{m} \Phi_{m}\left(\zeta_{\Phi_{m}}-\tau_{t}\right)=0 .
$$

As a result, the coefficient functions are

$$
\begin{gathered}
\tau\left(t, x, y, \Phi_{m}\right)=\frac{t^{2}}{2} C_{9}+t C_{8}+C_{1}, \\
\xi\left(t, x, y, \Phi_{m}\right)=\frac{t x}{2} C_{9}+\frac{x}{2} C_{8}+t C_{6}+y C_{4}+C_{2}, \\
\eta\left(t, x, y, \Phi_{m}\right)=\frac{t y}{2} C_{9}+\frac{y}{2} C_{8}+t C_{7}-x C_{4}+C_{3},
\end{gathered}
$$

where $C_{1}, C_{2}, C_{3}, C_{4}, C_{5}, C_{6}, C_{7}, C_{8}$ and $C_{9}$ are arbitrary constants and $\varpi(x, y, t)$ satisfies the following PDE

$$
\varpi_{t}-a_{m}\left(\varpi_{x x}+\varpi_{y y}\right)-b_{m} \varpi=0 .
$$

The infinitesimal generators is spanned by the vector field

$$
\begin{array}{r}
X_{1}=\frac{\partial}{\partial t}, \\
X_{6}=t \frac{\partial}{\partial x}-\frac{x}{2 a_{m} \Phi_{m}} \frac{\partial}{\partial \Phi_{m}}, \\
X_{2}=\frac{\partial}{\partial x}, \\
X_{3}=t \frac{\partial}{\partial y}-\frac{y}{2 a_{m} \Phi_{m}} \frac{\partial}{\partial \Phi_{m}}, \\
X_{8}=t \frac{\partial}{\partial t}+\frac{x}{2} \frac{\partial}{\partial x}+\frac{y}{2} \frac{\partial}{\partial y}+b_{m} t \Phi_{m} \frac{\partial}{\partial \Phi_{m}}, \\
X_{9}=t^{2} \frac{\partial}{\partial t}+t x \frac{\partial}{\partial x}+t y \frac{\partial}{\partial y}+\left(b_{m} t^{2}-t-\left(\frac{x^{2}+y^{2}}{4 a_{m}}\right)\right) \Phi_{m} \frac{\partial}{\partial \Phi_{m}},
\end{array}
$$$$
X_{4}=y \frac{\partial}{\partial x}-x \frac{\partial}{\partial y}
$$$$
X_{5}=\Phi_{m} \frac{\partial}{\partial \Phi_{m}}
$$

and

$$
X_{\varpi}=\varpi(x, y, t) \frac{\partial}{\partial \Phi_{m}}
$$

where $\varpi(x, y, t)$ is an arbitrary solution of Eq. (2). The $X_{1}, \ldots, X_{9}$ is closed under the Lie bracket. The commutation relations are shown in following table: 
Table 3. Commutation table.

\begin{tabular}{|c|c|c|c|c|c|c|c|c|c|}
\hline & $X_{1}$ & $X_{2}$ & $X_{3}$ & $X_{4}$ & $X_{5}$ & $X_{6}$ & $X_{7}$ & $X_{8}$ & $X_{9}$ \\
\hline$X_{1}$ & 0 & 0 & 0 & 0 & 0 & $X_{2}$ & $X_{3}$ & $X_{1}+b_{m} X_{3}$ & $X_{8}-1 \frac{X_{5}}{2}$ \\
\hline$X_{2}$ & 0 & 0 & 0 & $-X_{3}$ & 0 & $-\frac{X_{5}}{2 a_{m}}$ & 0 & $\frac{X_{2}}{2}$ & $X_{6}$ \\
\hline$X_{3}$ & 0 & 0 & 0 & $X_{2}$ & 0 & 0 & $-\frac{X_{5}}{2 a_{m}}$ & $\frac{X_{3}}{2}$ & $X_{7}$ \\
\hline$X_{4}$ & 0 & $X_{3}$ & $-X_{2}$ & 0 & 0 & $X_{7}$ & $-X_{6}$ & 0 & 0 \\
\hline$X_{5}$ & 0 & $0^{0}$ & 0 & 0 & 0 & 0 & 0 & 0 & 0 \\
\hline$X_{6}$ & $-X_{2}$ & $\frac{X_{5}}{2 a_{m}}$ & 0 & $-X_{7}$ & 0 & 0 & 0 & $-\frac{X_{6}}{2}$ & 0 \\
\hline$X_{7}$ & $-X_{3}$ & 0 & $\frac{X_{5}}{2 a_{m}}$ & $X_{6}$ & 0 & 0 & 0 & $-\frac{X_{7}}{2}$ & 0 \\
\hline$X_{8}$ & $\begin{array}{l}-X_{1} \\
-b_{m} X_{3}\end{array}$ & $-\frac{X_{2}}{2}$ & $-\frac{X_{3}}{2}$ & 0 & 0 & $\frac{X_{6}}{2}$ & $\frac{X_{7}}{2}$ & 0 & $X_{9}$ \\
\hline$X_{9}$ & $-X_{8}+\frac{X_{5}}{2}$ & $-X_{6}$ & $-X_{7}$ & 0 & 0 & 0 & 0 & $-X_{9}$ & 0 \\
\hline
\end{tabular}

The symmetry groups corresponding to the infinitesimal generators are found by solving the Lie equations

$$
\begin{gathered}
\frac{d \tilde{t}}{d \varepsilon}=\tau\left(\tilde{t}, \tilde{x}, \tilde{y}, \widetilde{\Phi}_{m}\right), \tilde{t}(\varepsilon=0)=t ; \\
\frac{d \tilde{x}}{d \varepsilon}=\xi\left(\tilde{t}, \tilde{x}, \tilde{y}, \widetilde{\Phi}_{m}\right), \tilde{x}(\varepsilon=0)=x ; \\
\frac{d \tilde{y}}{d \varepsilon}=\eta\left(\tilde{t}, \tilde{x}, \tilde{y}, \widetilde{\Phi}_{m}\right), \tilde{y}(\varepsilon=0)=y ; \\
\frac{d \widetilde{\Phi}_{m}}{d \varepsilon}=\zeta\left(\tilde{t}, \tilde{x}, \tilde{y}, \widetilde{\Phi}_{m}\right), \widetilde{\Phi}_{m}(\varepsilon=0)=\Phi_{m} .
\end{gathered}
$$

The one-parameter symmetry groups $G_{i}$ generated by the $X_{i}(i=1, \ldots, 9, \varpi)$ are:

- Translation group

$$
\begin{aligned}
& G_{1}: t+\varepsilon, x, y, \Phi_{m}, \\
& G_{2}: t, x+\varepsilon, y, \Phi_{m}, \\
& G_{3}: t, x, y+\varepsilon, \Phi_{m},
\end{aligned}
$$

- Rotation group

$$
G_{4}: t, x \cos (\varepsilon)+y \sin (\varepsilon), y \cos (\varepsilon)-x \sin (\varepsilon), \Phi_{m},
$$

- Scaling group

$$
\begin{gathered}
G_{5}: t, x, y, \Phi_{m} e^{\varepsilon} \\
G_{8}: t e^{2 \varepsilon}, x e^{\varepsilon}, y e^{\varepsilon}, \Phi_{m} \exp \left(b_{m} t\left(e^{2 \varepsilon-1}\right)\right),
\end{gathered}
$$

- Classical Galilean group

$$
\begin{gathered}
G_{6}: t, x+t \varepsilon, y, \Phi_{m} \exp \left(-\frac{t \varepsilon^{2}+2 x \varepsilon}{4 a_{m}}\right), \\
G_{7}: t, x, y+t \varepsilon, \Phi_{m} \exp \left(-\frac{t \varepsilon^{2}+2 y \varepsilon}{4 a_{m}}\right),
\end{gathered}
$$

- Projection group

$$
\begin{gathered}
G_{9}: \frac{t}{1-t \varepsilon}, \frac{x}{1-t \varepsilon}, \frac{y}{1-t \varepsilon}, \Phi_{m}(1-t \varepsilon) \\
\exp \left(-\frac{t \varepsilon}{1-t \varepsilon}\left(b_{m} t-\frac{x^{2}+y^{2}}{4 a_{m} t}\right)\right),
\end{gathered}
$$

- and the infinite-dimensional system group

$$
G_{\varpi}: t, x, y, \Phi_{m}+\varpi(x, y, t) \varepsilon .
$$

\section{Invariant Solutions}

In the previous section, the symmetry groups are obtained. Now, this section considers the invariant solutions of the MNDE based on the symmetry analysis. Since each $G_{i}(i=$ $1, \ldots, 9)$ is a symmetry group, it implies that $\Phi_{m}=$ $f(x, y, t)$ is a solution of Eq. (2) [6-8]. As a result, the infinitesimal generator $X$ exists with an invariant function $f$

$$
X f=0 \Rightarrow \tau \frac{\partial f}{\partial t}+\xi \frac{\partial f}{\partial x}+\eta \frac{\partial f}{\partial y}+\zeta \frac{\partial f}{\partial \Phi_{m}}=0
$$

The invariant function is obtained by integrating the characteristic equation:

$$
\frac{d t}{\tau}=\frac{d x}{\xi}=\frac{d y}{\eta}=\frac{d \Phi_{m}}{\zeta} .
$$

Indeed, all invariant solutions are invariant under the action of the Lie group.

1. The characteristic equations corresponding to the translation group are:

- For the generator $X_{1}$,

$$
\frac{d t}{1}=\frac{d x}{0}=\frac{d y}{0}=\frac{d \Phi_{m}}{0} .
$$

- For the generator $X_{2}$,

$$
\frac{d t}{0}=\frac{d x}{1}=\frac{d y}{0}=\frac{d \Phi_{m}}{0} .
$$

- For the generator $X_{3}$,

$$
\frac{d t}{0}=\frac{d x}{0}=\frac{d y}{1}=\frac{d \Phi_{m}}{0} .
$$

The similarity variables are

$$
\begin{aligned}
& g=t ; f(y)=\Phi_{m}, \\
& g=x ; f(x)=\Phi_{m},
\end{aligned}
$$




$$
g=y ; f(t)=\Phi_{m} .
$$

The case of $\Phi_{m}=f(y)$ or $\Phi_{m}=f(x)$ reduces the Eq. (2) to the ordinary differential equation (ODE)

$$
f^{\prime \prime}+\frac{b_{m}}{a_{m}} f=0
$$

with $f^{\prime}=d f / d y$ and $f^{\prime}=d f / d x$.

By solving the ODE, the invariant solution is

$$
\begin{aligned}
& \Phi_{m}(x, y, t)=c_{1} \sin \left(\sqrt{\frac{b_{m}}{a_{m}}} y\right)+c_{1}^{\prime} \cos \left(\sqrt{\frac{b_{m}}{a_{m}}} y\right), \\
& \Phi_{m}(x, y, t)=c_{2} \sin \left(\sqrt{\frac{b_{m}}{a_{m}}} x\right)+c_{2}^{\prime} \cos \left(\sqrt{\frac{b_{m}}{a_{m}}} x\right),
\end{aligned}
$$

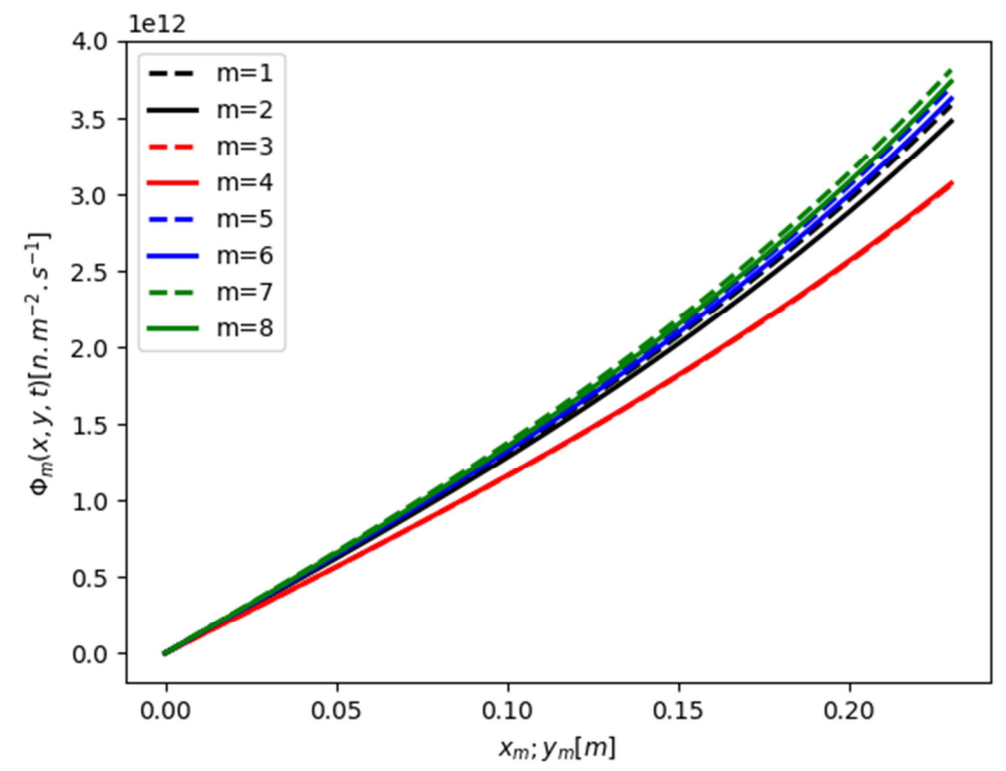

Figure 1. Neutron fluxes from time translation, with $c_{1}=c_{2}=\Phi_{m}^{0} / 4$ and $c_{1}^{\prime}=c_{2}^{\prime}=0$.

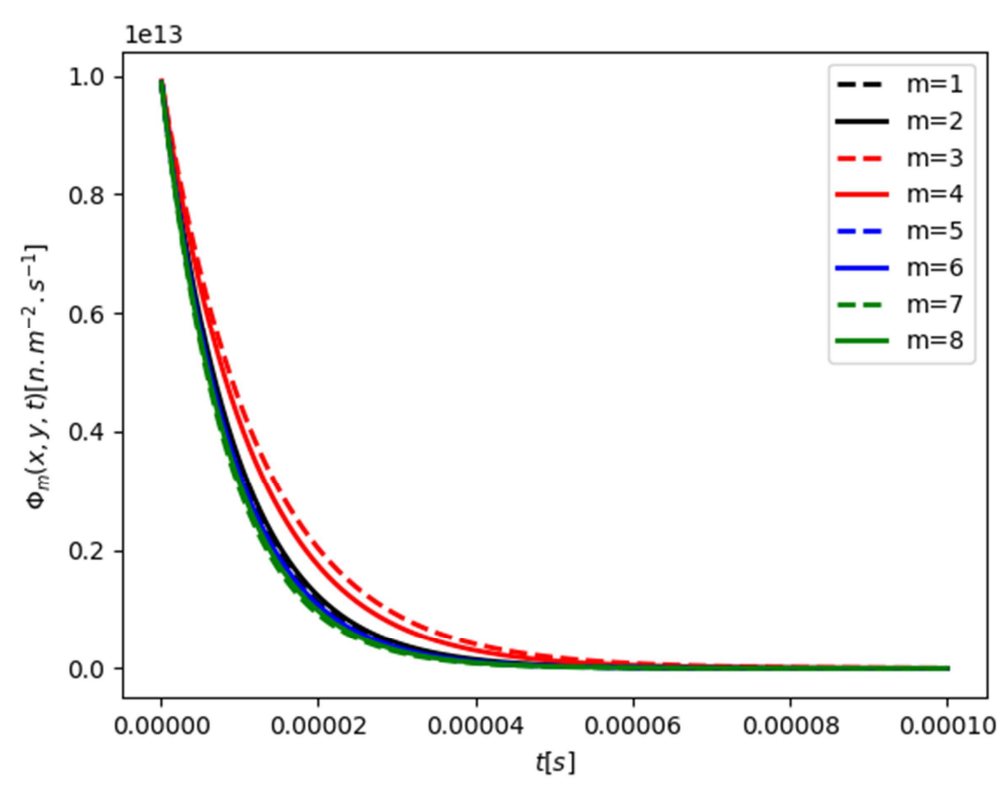

Figure 2. Neutron fluxes from space translation, with $c_{3}=\Phi_{m}^{0}$.

The solutions (46) and (47) correspond to the first type of steady-state solution of Eq. (2). The plot of each region has the same shape evolving in space. It means that as the width of each region is reached, then the fluxes with respect to $x_{m}$ and $y_{m}$ increase their descriptive values. ODE

$$
f^{\prime}-b_{m} f=0
$$

with $f^{\prime}=d f / d t$.

By solving the ODE, the invariant solution is

$$
\Phi_{m}(x, y, t)=c_{3} \exp \left(b_{m} t\right)
$$


where $c_{3}$ is the integration constant.

The solution (49) presents the property of the kinetic model without delayed neutrons and external source. The plot shows the evolution of fluxes by regions with respect to time. The fluxes decrease as soon as the time increases in value and become null in every point of the regions. If $b_{m}$ takes a positive value, the output of the neutron flux will be greater than the value of the initial flux $\Phi_{m}^{0}$ average, so there will be a bifurcation at the level of the concerned region.

2. The characteristic equation of the generator $X_{4}$ corresponding to the rotation group is:

$$
\frac{d t}{0}=\frac{d x}{y}=\frac{d y}{-x}=\frac{d \Phi_{m}}{0}
$$

The similarity variable is

$$
g=\sqrt{x^{2}+y^{2}} ; f(g)=\Phi_{m} .
$$

The Eq. (2) is reduced to the ODE in the form of Bessel's equation

$$
f^{\prime \prime}+\frac{1}{g} f^{\prime}+\frac{b_{m}}{a_{m}} f=0
$$

with $f^{\prime}=d f / d g$.

By solving the Bessel's equation, the invariant solution is

$$
\Phi_{m}(x, y, t)=c_{4} J_{0}\left(\sqrt{\frac{b_{m}}{a_{m}}} \sqrt{x^{2}+y^{2}}\right)+c_{4}^{\prime} Y_{0}\left(\sqrt{\frac{b_{m}}{a_{m}}} \sqrt{x^{2}+y^{2}}\right),
$$

where $c_{4}$ and $c_{4}^{\prime}$ are the integration constants.

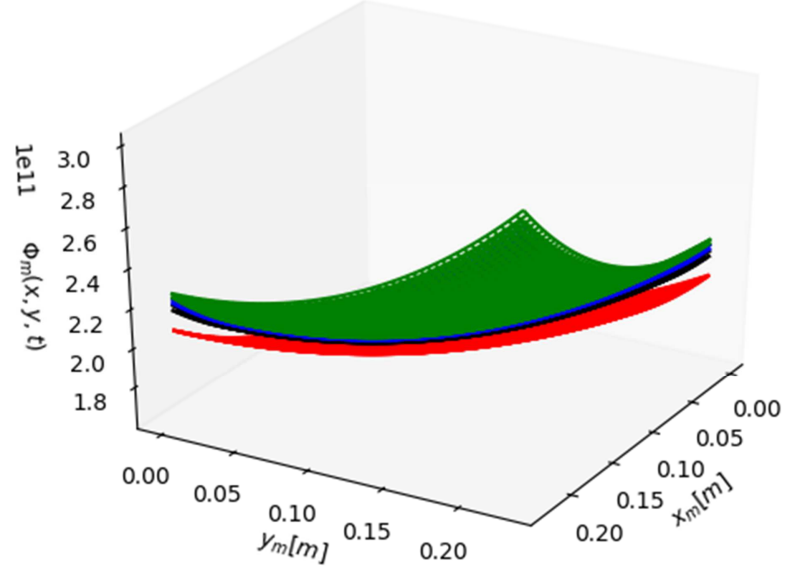

Figure 3. Neutron fluxes from rotation group, with $c_{4}=\Phi_{m}^{0} / 2 \pi^{3}$ and $c_{4}^{\prime}=$

$J_{0}$ and $Y_{0}$ are Bessel functions [11]. This result corresponds to the second type of steady-state of Eq. (2), for the higher value of the flux by the term $\sqrt{b_{m}} / a_{m} \sqrt{x^{2}+y^{2}}$. The plot of these fluxes is characteristic of rotation in the plane.

The neutron fluxes are therefore distributed throughout the plane of the regions with maximum value.

3. The characteristic equations corresponding to the classical Galilean group are:

- For the generator $X_{6}$,

$$
\frac{d t}{0}=\frac{d x}{t}=\frac{d y}{0}=\frac{-2 a_{m} d \Phi_{m}}{x \Phi_{m}} .
$$

- For the generator $X_{7}$,

$$
\frac{d t}{0}=\frac{d x}{0}=\frac{d y}{t}=\frac{-2 a_{m} d \Phi_{m}}{y \Phi_{m}} .
$$

The similarity variables are

$$
g=t ; f(g)=\Phi_{m} \exp \left(\frac{x^{2}}{4 a_{m} t}\right),
$$

$$
\begin{gathered}
g=t ; f(g)=\Phi_{m} \exp \left(\frac{y^{2}}{4 a_{m} t}\right), \\
g=t ; f(g)=\Phi_{m} \exp \left(\frac{x^{2}+y^{2}}{4 a_{m} t}\right) .
\end{gathered}
$$

The case of $\Phi_{m}=e^{-\frac{x^{2}}{4 a_{m} t}} f(t) \quad$ or $\quad \Phi_{m}=$ $e^{-\frac{y^{2}}{4 a_{m} t}} f(t)$ reduces the Eq. (2) to the following ODE

$$
f^{\prime}+\left(\frac{1}{2 t}-b_{m}\right) f=0
$$

with $f^{\prime}=d f / d t$.

By solving the ODE, the invariant solutions are

$$
\begin{aligned}
& \Phi_{m}(x, y, t)=c_{6} t^{-\frac{1}{2}} \exp \left(b_{m} t-\frac{x^{2}}{4 a_{m} t}\right), \\
& \Phi_{m}(x, y, t)=c_{7} t^{-\frac{1}{2}} \exp \left(b_{m} t-\frac{y^{2}}{4 a_{m} t}\right),
\end{aligned}
$$

where $c_{6}$ and $c_{7}$ are the integration constants.

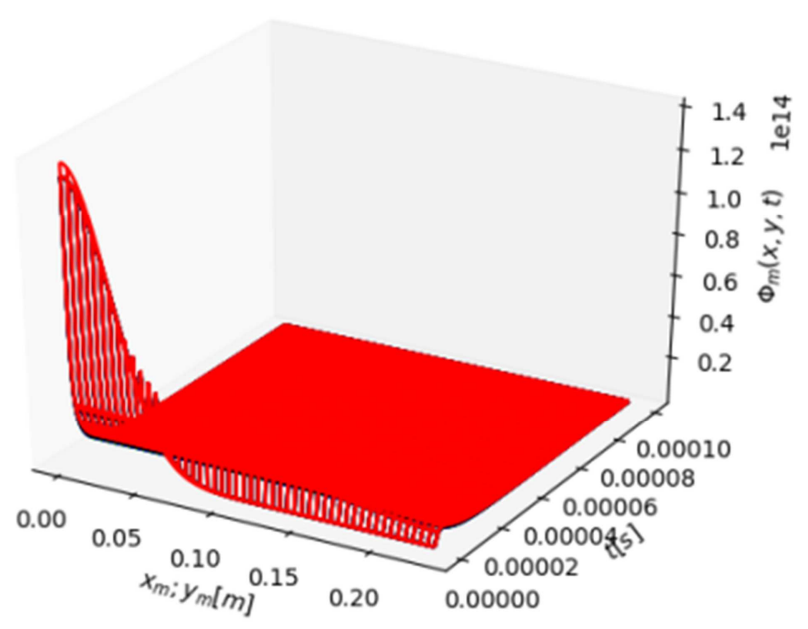

Figure 4. Neutron fluxes from classical Galilean group with respect to $x_{m}$ or $y_{m}$, with $c_{6}=c_{7}=\Phi_{m}^{0} / \sqrt{4 \pi a_{m}}$.

The solutions (53) and (54) represent at first the kinetic 
model part

$$
\Phi_{m}^{0} \exp \left(b_{m} t\right)
$$

and the diffusion by random walk distribution with respect to $(x, y)$

$$
\begin{aligned}
& \frac{\Phi_{m}^{0}}{\sqrt{4 \pi a_{m} t}} \exp \left(-\frac{x^{2}}{4 a_{m} t}\right), \\
& \frac{\Phi_{m}^{0}}{\sqrt{4 \pi a_{m} t}} \exp \left(-\frac{y^{2}}{4 a_{m} t}\right),
\end{aligned}
$$

on the interval $\left[-x_{m} / 2,+x_{m} / 2\right]$ and $\left[-y_{m} / 2,+y_{m} / 2\right]$. This part of solutions characterizes the random collision of neutrons with the fuel material.

The case of $\Phi_{m}=e^{-\frac{x^{2}+y^{2}}{4 a_{m} t}} f(t)$ reduces the Eq. (2) to the following ODE

$$
f^{\prime}+\left(\frac{1}{t}-b_{m}\right) f=0
$$

with $f^{\prime}=d f d t$.

By solving the first order ODE, the invariant solution is

$$
\Phi_{m}(x, y, t)=c_{67} t^{-1} \exp \left(b_{m} t-\frac{x^{2}+y^{2}}{4 a_{m} t}\right),
$$

where $c_{67}$ is the integration constant.

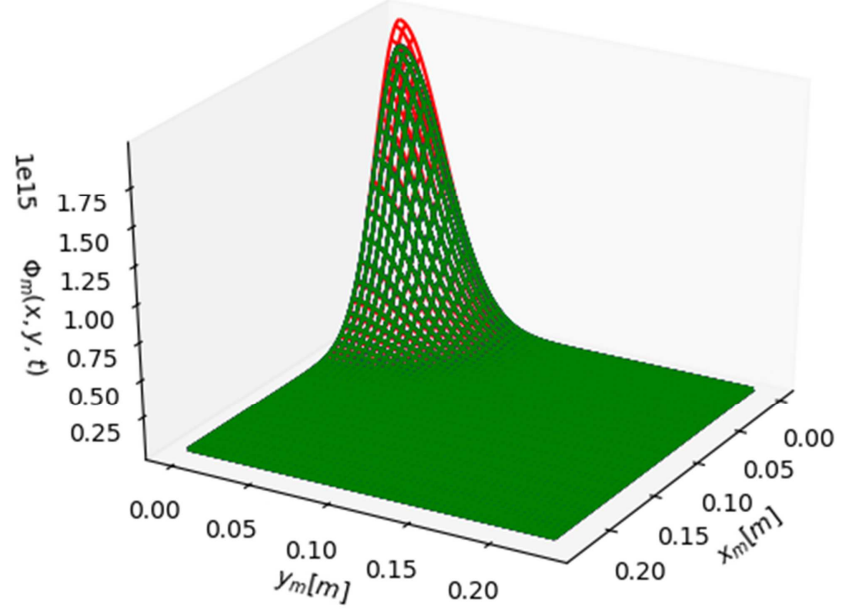

Figure 5. Neutron fluxes from classical Galilean group with respect to $x_{m}$ and $y_{m}$, with $c_{67}=\Phi_{m}^{0} / 4 \pi a_{m}$.

Excluding the kinetic model, the two-dimensional random walk diffusion term is

$$
\frac{\Phi_{m}^{0}}{4 \pi a_{m} t} \exp \left(-\frac{x^{2}+y^{2}}{4 a_{m} t}\right)
$$

on the surface of regions $\left[-x_{m} / 2,+x_{m} / 2\right] \times\left[-y_{m} /\right.$ $\left.2,+y_{m} / 2\right]$. The neutron fluxes move in the plane of the regions, regardless of the nature of the material compositions. The probability of locating neutrons is more precise in the same plane $(x, y)$ at the instant $t$ whatever and that the existence of collisions is more probably.
4. The characteristic equation for generator $X_{8}$ from scaling group is

$$
\frac{d t}{t}=2 \frac{d x}{x}=2 \frac{d y}{y}=\frac{d \Phi_{m}}{b_{m} t \Phi_{m}} .
$$

The similarity variables are

$$
\begin{gathered}
g=\frac{x}{\sqrt{t}} ; f(g)=\Phi_{m} \exp \left(-b_{m} t\right), \\
g=\frac{y}{\sqrt{t}} ; f(g)=\Phi_{m} \exp \left(-b_{m} t\right), \\
g=\frac{x^{2}+y^{2}}{t} ; f(g)=\Phi_{m} \exp \left(-b_{m} t\right) .
\end{gathered}
$$

The case of $\Phi_{m}=f(x / \sqrt{t}) e^{b_{m} t}$ or $\Phi_{m}=f(y /$ $\sqrt{t}) e^{b_{m} t}$ reduces the Eq. (2) to the following ODE

$$
f^{\prime}+\frac{g}{2 a_{m}} f=0
$$

with $f^{\prime}=d f / d g$.

By solving the ODE, the invariant solutions are

$$
\begin{aligned}
& \Phi_{m}(x, y, t)=\left(\operatorname{erf}\left(\frac{x}{2 \sqrt{a_{m} t}}\right) c_{8}+c_{8}^{\prime}\right) \exp \left(b_{m} t\right), \\
& \Phi_{m}(x, y, t)=\left(\operatorname{erf}\left(\frac{y}{2 \sqrt{a_{m} t}}\right) k_{8}+k_{8}^{\prime}\right) \exp \left(b_{m} t\right),
\end{aligned}
$$

where $c_{8}, c_{8}^{\prime}, k_{8}$ and $k_{8}^{\prime}$ are the integration constants.

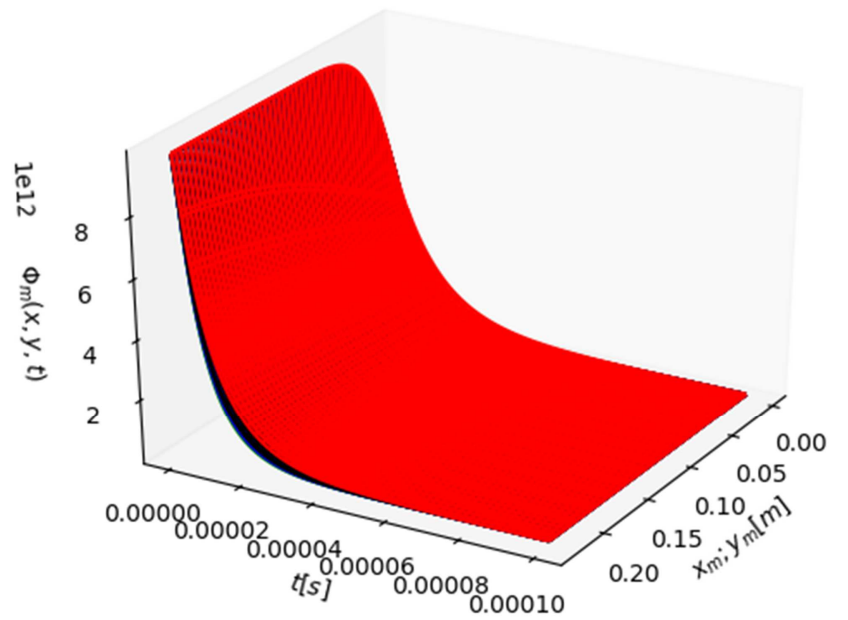

Figure 6. Neutron fluxes from scaling group, with $c_{8}=c_{8}^{\prime}=k_{8}=k_{8}^{\prime}=$ $\Phi_{m}^{0} / 2$.

The solutions (58) and (59) represent the kinetic model and the functions

$$
\begin{aligned}
& \frac{\Phi_{m}^{0}}{2}\left(1+\operatorname{erf}\left(\frac{x}{2 \sqrt{a_{m} t}}\right)\right), \\
& \frac{\Phi_{m}^{0}}{2}\left(1+\operatorname{erf}\left(\frac{y}{2 \sqrt{a_{m} t}}\right)\right) .
\end{aligned}
$$

The function $\operatorname{erf}\left(y_{m} / 2 \sqrt{a_{m} t}\right)$ or $\operatorname{erf}\left(x_{m} / 2 \sqrt{a_{m} t}\right)$ related 
to the cumulative distribution $\Phi_{m}(x, y, t)$ expresses the normal distribution of neutron fluxes for the variable $y /$ $2 \sqrt{a_{m} t}$ or $x / 2 \sqrt{a_{m} t}$ of the diffusion equation and favorable to a fission reaction condition.

The case of $\Phi_{m}=f\left(\left(x^{2}+y^{2}\right) / t\right) e^{b_{m} t}$ reduces the Eq. (2) to the following ODE

$$
f^{\prime \prime}+\left(\frac{1}{4 a_{m}}+\frac{1}{g}\right) f^{\prime}=0,
$$

With $f^{\prime}=d f / d g$.

By solving the second-order of ODE, the invariant solution is

$$
\Phi_{m}(x, y, t)=\left(E_{1}\left(\frac{x^{2}+y^{2}}{4 a_{m} t}\right) q_{8}+q_{8}^{\prime}\right) \exp \left(b_{m} t\right) .
$$

Where $q_{8}$ and $q_{8}^{\prime}$ are the integration constants.

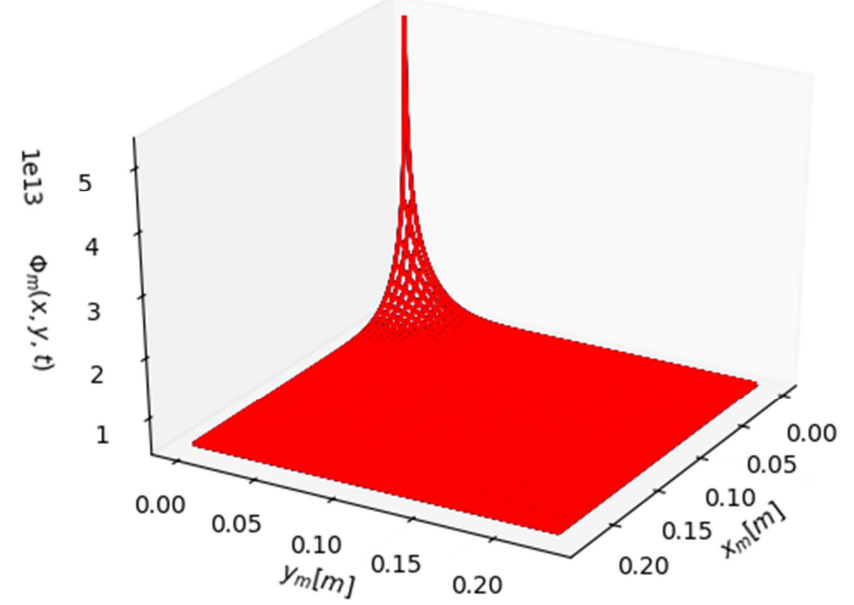

Figure 7. Neutron fluxes from scaling group, with $q_{8}=q_{8}^{\prime}=\Phi_{m}^{0} / 2$.

$E_{1}$ represents first order integral exponential function of $\left(x^{2}+y^{2}\right) / 4 a_{m} t$. Excluding the kinetic model, the solution (61) has the function

$$
\frac{\Phi_{m}^{0}}{2}\left(1+E_{1}\left(\frac{x^{2}+y^{2}}{4 a_{m} t}\right)\right)
$$

that represents the uncollided neutron fluxes with the fuel material [1], in opposition of the solutions (53), (54) and (56).

5. The characteristic equation of generator $X_{9}$ from projection group is

$$
\frac{d t}{t^{2}}=\frac{d x}{t x}=\frac{d y}{t y}=\frac{d \Phi_{m}}{\left(b_{m} t^{2}-t-\frac{x^{2}+y^{2}}{4 a_{m}}\right) \Phi_{m}} .
$$

The similarity variable is

$$
g=\frac{x^{2}+y^{2}}{t^{2}} ; f(g)=\Phi_{m} t \exp \left(-b_{m} t+\frac{x^{2}+y^{2}}{4 a_{m} t}\right) \text {. }
$$

The case of $\Phi_{m}=f(g) t^{-1} e^{b_{m} t-(x 2+y 2) / 4 a_{m} t}$ reduces the Eq. (2) to the following ODE

$$
f^{\prime \prime}+\frac{1}{g} f^{\prime}=0
$$

with $f^{\prime}=d f / d g$.

By solving the ODE, the invariant solution is

$$
\Phi_{m}(x, y, t)=\frac{c_{9}}{t}+\frac{c_{9}^{\prime}}{t} \ln \left(\frac{x^{2}+y^{2}}{t^{2}}\right) \exp \left(b_{m} t-\frac{x^{2}+y^{2}}{4 a_{m} t}\right),
$$

where $c_{9}$ and $c_{9}^{\prime}$ are the integration constants.

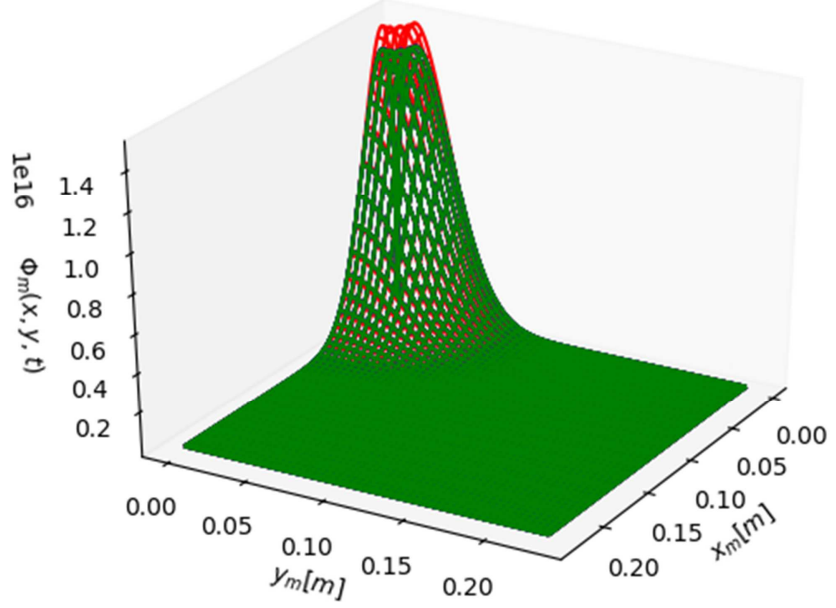

Figure 8. Neutron fluxes from projection group, with $c_{9}=c_{9}^{\prime}=\Phi_{m}^{0} / 4 \pi a_{m}$.

The solution (59) represents the kinetic model and the projection of solution (56) for the random walk on the plan $(x, y)$

$$
\frac{\Phi_{m}^{0}}{4 \pi a_{m} t}\left(1+\ln \left(\frac{x^{2}+y^{2}}{t^{2}}\right)\right) \exp \left(b_{m} t-\frac{x^{2}+y^{2}}{4 a_{m} t}\right) .
$$

The term

$$
\frac{\Phi_{\mathrm{m}}^{0}}{4 \pi a_{m} t} \ln \left(\frac{x^{2}+y^{2}}{t^{2}}\right) \exp \left(b_{m} t-\frac{x^{2}+y^{2}}{4 a_{m} t}\right) .
$$

represents the projected part of the solution.

To the steady-state solution, the case for $m=7$ where the neutrons flux is more remarkable having a higher value than the other regions. Whereas for $m=3$, the value of the flux is small compared to the other regions, so has a neutron flux easily absorbed, well

$$
M=3<4<1<2<6<5<8<7 .
$$

To the kinetic model and its influence, $m=3$ represents the maximum value per region, the regional flux corresponding to the fast decay of reactor relative to other regions. There is no possibility of fission but only absorption.

The value decreases by region and beyond this period $\left(t=10^{-4} s\right)$ the flux tends to 0 . The linear plots become flat. On the other hand, the region $m=7$ has a slow decay having a lower value than the other. In this case, a large possibility of fission reaction and a weak absorption rate are remarked. Intermediate regions in both regions $(m=3,7)$ retain the appearance of Figure 2 


$$
M=3>4>1>2>6>5>8>7 .
$$

The distribution of neutrons produced by collisions occurs in the media. Its dependence on space is determined by the properties of the media, except for the normal distribution, and its dependence on time shows the evolution. The system therefore represents a situation in which the collisional state exists.

On the other part, the distribution gives the departure of the collisional state flux caused by the fissile material. As a result, the material and the properties of the media affect the flux.

\section{Conclusions}

The symmetry groups related to one-parameter are obtained and the MNDE are solved from Lie symmetry group in order to have several invariant solutions representing each of them the sense of diffusion. These explicit solutions for Cartesian geometry are examined that are given for the first time in this work and had characteristics that might be important for the nuclear reactor physics.

However, the case of the generator $X_{5}$ gives a trivial solution at $t=0$

$$
\Phi_{m}(x, y, t)=c_{5}=\Phi_{m}^{0},
$$

which illustrates the initiator flux of the chain reaction.

The neutron coefficients of the 2D BIBLIS Benchmark have been used in order to illustrate behavior of the system that occur significant branch.

The 2D BIBLIS benchmark has demonstrated the ability of solutions to multiple regions. The neutron's behavior is affected by the diffusion effects, boundary conditions and surface to determine the integration constants. The neutron flux profiles are plotted through several graphs for various values of the emerging parameters. Each plot has shown the integrated system fluxes of the 8 regions. In most of solutions, we found that the kinetic model is a very important part for any form of solutions generated by symmetries groups. The solutions were averaged at times $t=$ $10^{-7} s, 10^{-6} s, 10^{-5} s$ and $10^{-4} s$.

The observations concerning the validity of the multiregion solution in relation to the relevance of diffusion in the characterization of neutron fluxes behavior for reactor conditions lead to contributions to mentioned solutions.

The two-dimensional mono-kinetic MNDE has been used to explore the neutron behavior in Cartesian model.

\section{References}

[1] Bell, George I. and Glasstone Samuel: "Nuclear Reactor Theory", Van Nostrand Reinhold, 1970.

[2] James J. Duderstadt, Louis J. Hamilton: "Nuclear Reactor Analysis", Department of Nuclear Engineering, University of Michigan, 1976.

[3] R. A. Shober: "A Nodal Method For solving Transient Fewgroup Neutron Diffusion Equation", Applied Physics Division, Argonne National Laboratory, pp 11-17, June 1978.

[4] Alain Hebert: "Multigroup Neutron Tranport and Diffusion Computations", Handbook of Nuclear Engineering, Vol. 2, Editor: Dan Gabriel Cacuci, pp 753-911, 2010.

[5] Joe. W. Durkee Jr: "Exact solution to the time-dependent onespeed multiregion neutron diffusion equation", Progress in Nuclear Energy, Volume 3, No 3, pp 191-222, 1994.

[6] Ovsyannikov L. V.: "Group Analysis of Differential Equations”, New York Academic Press, 1982.

[7] Peter J. Olver: "Applications of Lie Groups to Differential Equations", Springer - Verlag, New York, Grad. Texts in Math. 107, 1986.

[8] Nail H. Ibragimov: "CRC Handbook of Lie Group Analysis of Differential Equations", Vol. 1: Symmetries, Exact Solutions and Conservation Laws, CRC Press, Boca Raton, 1994.

[9] Brian Christensen: "Three-dimensional Static and Dynamic Reactor Calculations by the Nodal Expansion Method", Risø National Laboratory, DK-4000 Roskilde, Denmark, 1985.

[10] E. Z. Muller and Z. J. Weiss: "Benchmarking with the multigroup diffusion high-order response matrix method", Annals of Nuclear Energy, vol. 18, no. 9, pp. 535-544, 1991.

[11] Ivan Tsyfra and Tomasz Czyżycki: "Symmetry and Solution of Neutron Transport Equations in Nonhomogeneous Media", Hindawi Publishing Corporation, Volume 2014, Article ID 724238, 17 June 2014.

[12] Seyed Abolfazl Hosseini: "Sensitivity Analysis of the Galerkin Finite Element Method Neutron Diffusion Solver to the Shape of the Elements", Nuclear Engineering and Technology 49 (2017), 29-42, 8 August 2016.

[13] Seyed Abolfazl Hosseini and Farahnaz SaadatianDerakhshande: "Galerkin and Generalized Least Squares finite element: A comparative study for multi-group diffusion solvers", Progress in Nuclear Energy 85, 473-490, 20 July 2015.

[14] Sukanta Nayak and S. Chakraverty: "Numerical Solution of two group uncertain neutron diffusion equation for multiregion reactor", Third International Conference on Advances in Control and Optimization of Dynamical Systems, Kanpur, India, March 13-15, 2014. 